Teil 2

Anwendungsfälle und Einsatzgebiete von »Fake News« 


\section{Neue Debatte, altes Dilemma? \\ Die Herausforderungen des Phänomens »Fake News« für die Unternehmenskommunikation}

Michael Johann \& Jana Wagner

Abstract

Die Verbreitung von Falschinformationen über die sozialen Medien und das erodierende Vertrauen in Teilen der Gesellschaft belasten nicht nur den Journalismus und die politische Kommunikation, sondern auch die strategische Kommunikation von Unternehmen. Vertrauensbedrohliche Phänomene wie "Fake News" stellen jedoch keine genuin neuen Herausforderungen für die Unternehmenskommunikation dar, sondern erfordern im Grunde eine Rückbesinnung auf klassische Bezugsgrößen der Public Relations (PR) wie Legitimation, Vertrauen und Glaubwürdigkeit. Ausgehend von einer begrifflichen und konzeptuellen Unterscheidung zwischen Fehlinformationen, Falschinformationen und schädigenden Informationen entwickelt der vorliegende Beitrag anhand von Fallbeispielen aus der Kommunikationspraxis vier Risikoszenarien, welche die Relevanz des Phänomens »Fake News " für die Unternehmenskommunikation vor allem auf der Makro- und der Mesoebene systematisch erfassen. Die Szenarien verdeutlichen, dass die Grenzziehung zwischen Wahrheit und Unwahrheit, PR-Gag und gezielter Fehlleitung schwierig sein kann und daher angesichts der aktuellen gesellschaftlichen Debatte rund um das Phänomen "Fake News" besondere Achtsamkeit seitens der Unternehmenskommunikation gefordert ist.

\section{Das Dilemma der Unternehmenskommunikation}

Das Phänomen »Fake News« im Sinne intentional konstruierter Falschmeldungen, die aus ökonomischen oder ideologischen Gründen erfunden werden (Arendt, Haim \& Beck, 2019), bergen nicht nur für den Journalismus, sondern auch für die Unternehmenskommunikation erhebliche Risiken (Kirf, 2018). Unternehmenskommunikation wird generell verstanden als »alle gesteuerten Kommunikationsprozesse, mit denen ein Beitrag zur 
Aufgabendefinition und -erfüllung in gewinnorientierten Wirtschaftseinheiten geleistet wird und die insbesondere zur internen und externen Handlungskoordination sowie Interessenklärung zwischen Unternehmen und ihren Bezugsgruppen (Stakeholdern) beitragen« (Zerfaß, 2014, 23). Als Aufgabenfeld der Unternehmenskommunikation ist es vor allem das Ziel der Public Relations (PR), das Vertrauen relevanter Stakeholder in das Unternehmen und die eigene Glaubwürdigkeit zu pflegen. Die aktuell beobachtbare Erosion des Vertrauens verschiedener Teile der Gesellschaft in Medien, Unternehmen, Regierungen und NGOs (Edelman, 2019; FleishmanHillard, 2018; Jackob et al., 2019; Ziegele et al., 2018) steht diesem Ziel jedoch diametral entgegen und macht Unternehmen grundsätzlich anfälliger für die vielschichtigen Gefahren durch »Fake News« wie beispielsweise Glaubwürdigkeits- und Reputationsverluste.

So geben zahlreiche Unternehmen in Europa (Zerfaß, Tench, Verhoeven, Verčič \& Moreno, 2018) und Nordamerika (Meng, Reber, Berger, Gower \& Zerfaß, 2019) an, von der Verbreitung von »Fake News« durch interne Kommunikationskanäle, traditionelle Massenmedien und vor allem soziale Medien betroffen zu sein. Die European Association of Communication Directors (2017) appelliert in einem Manifest gegen »Fake News« angesichts des zunehmenden Vertrauensverlustes an die ethischen Grundsätze des PRHandelns. Mit dem Code d'Athènes, dem Code of Lisbon, dem Global Protocol on Ethics in Public Relations oder dem IPRA Code of Conduct stehen der Kommunikationspraxis dabei verschiedene international anerkannte Ethik-Kodizes zur Verfügung, an denen sich die Unternehmenskommunikation messen muss. Speziell in Deutschland gilt der Deutsche Kommunikationskodex des Deutschen Rates für Public Relations (DRPR), der mit Mahnungen und Rügen entsprechende Verstöße regelmäßig sanktioniert.

Betrachtet man den aktuellen Forschungsdiskurs, zeigt sich, dass das Phänomen »Fake News« vor allem in Verbindung mit Herausforderungen für den Journalismus und die politische Kommunikation diskutiert wird (unter anderem Allcott \& Gentzkow, 2017; Arendt et al., 2019; Brummette, DiStaso, Vafeiadis \& Messner, 2018; Corner, 2017; Gunther, Beck \& Nisbet, 2019; Ireton \& Posetti, 2018; Müller \& Denner 2017). In der PRForschung ist Zurückhaltung bei der Verwendung des Begriffes zu beobachten. Dabei gilt der Bereich PR/Werbung nach der vielzitierten Typologie von Tandoc, Lim \& Ling (2018) sogar als eine eigenständige Ausprägung von »Fake News«. So handle es sich in diesem Kontext um »Fake News «, »when public relations practitioners adopt the practices and/or appearance of journalists in order to insert marketing or other persuasive messages into news media« (Farsetta \& Price, 2006, 5; Tandoc et al., 2018, 
145). Die genauere Betrachtung dieses Definitionsversuches zeigt, dass in der aktuellen Debatte um das Phänomen »Fake News« im Grunde ein klassisches Dilemma der Unternehmenskommunikation angesprochen wird: die wechselseitige Beziehung zwischen PR und Journalismus (Baerns, 1985; Bentele, Liebert \& Seeling, 1997) und damit die Frage nach der normativen Leistung von PR im Grenzbereich zwischen Unternehmen und gesellschaftlicher Umwelt (Hoffjann, 2009; Johann, 2020; Röttger, Preusse \& Schmitt, 2014).

Das Ziel dieses Beitrages ist es folglich, vor dem Hintergrund dieses augenscheinlichen Dilemmas, die Relevanz des Phänomens »Fake News « für die Unternehmenskommunikation auf der Meso- und Makroebene genauer zu erschließen. Hierzu erfolgt zunächst im zweiten Abschnitt eine Bestimmung und Abgrenzung von zentralen Begriffen, um den stark politisierten Begriff »Fake News« (Brummette et al., 2018) aus Sicht der Unternehmenskommunikation operational erfassen zu können. Der dritte Abschnitt widmet sich anschließend unterschiedlichen Fallbeispielen aus der Kommunikationspraxis, um die verschiedenen Szenarien und deren Relevanz zu illustrieren. Schließlich erfolgt im vierten Abschnitt eine Systematisierung in Form einer Risikomatrix, die sowohl für die Kommunikationspraxis als auch für PR-Forschung als systematische Annäherung an das Phänomen $»$ Fake News« dienen soll.

\section{Fehlinformationen, Falschinformationen, schädigende Informationen}

Vertrauensbedrohliche Phänomene wie »Fake News« stellen keine neuen Herausforderungen für die Unternehmenskommunikation dar. Der Handlungsbedarf, der damit einhergeht, ist eher als eine Renaissance klassischer Handlungsmaximen und Bezugsgrößen der PR zu werten, wie etwa Legitimation, Vertrauen, Glaubwürdigkeit, Image, Reputation oder Verständigungsorientierung. Gerüchte, Halbwahrheiten oder Verschleierungsversuche sind genuine Grauzonen der kommunikativen Praxis, wenn es um die optimierende Darstellung eines Unternehmens im Sinne der Gewährleistung dieser Bezugsgrößen geht - ihnen wird mitunter sogar funktionaler Nutzen für Individuen, Gruppen und Unternehmen zugeschrieben (Mast, 2019). PR steht jedoch - anders als Werbung - oftmals in der Kritik, ihre eigentlichen Absichten unter dem Vorwand der Gemeinwohlorientierung zu verschleiern (Habermas, 1990).

In der PR-Forschung wurde dieses Spannungsfeld vor allem durch das Konzept der Täuschung erfasst (Dulek \& Campbell, 2015; Merten, 2010; 
Thummes, 2013). Demnach handelt es sich um eine Täuschung, »wenn in einer Kommunikationssituation eine unwahrhaftige Handlung auftritt, die in der Absicht getätigt wird, andere in die Irre zu führen « (Thummes, 2016, 361). Thummes (2013) unterscheidet allgemein zwischen einer Schutzfunktion und einer Dominanzfunktion, die an kollaborative, altruistische und egoistische Motive gekoppelt sind. Mit dem Fokus auf die Intentionalität dieser kommunikativen Handlungen offenbaren sich starke Parallelen zu aktuellen Definitionen von »Fake News« (Arendt et al., 2019; Gelfert, 2018; Tandoc et al., 2018; Zimmermann \& Kohring, 2018; Zimmermann \& Kohring, in diesem Band). Thummes $(2016,361)$ schließt dabei allerdings unintentionale Handlungen grundsätzlich aus: »Handelt ein Akteur [...] mit Bezug auf unvorhersehbare zukünftige Entwicklungen oder gänzlich unwissend [...], gilt sein Verhalten nicht als Täuschung.« In diesem Beitrag soll jedoch mit Blick auf die Relevanz des Phänomens »Fake News« in der Unternehmenskommunikation ein breiteres Verständnis zugrunde gelegt werden, das unbewusste und bewusste Täuschungen gleichermaßen berücksichtigt.

Während im Deutschen noch um die passenden Begriffsbezeichnungen und Definitionen gerungen wird (Kohring \& Zimmermann, 2019; Scholl \& Völker, 2018; Zimmermann \& Kohring, 2018; Zimmermann \& Kohring, in diesem Band), konnte sich im englischen Sprachraum die Unterscheidung zwischen Misinformation und Disinformation etablieren (Karlova \& Fisher, 2012). In ihrem Modell der Information Disorder, das vor allem Eigenschaften der Sender, der Informationen selbst und der Empfänger betrachtet, ergänzen Wardle und Derakhshan (2018) neben Misinformation und Disinformation mit Malinformation eine dritte Ausprägung, welche die Schadensabsicht berücksichtigt. Dieses Modell soll als Grundlage für die folgende Erschließung von »Fake News« in der Unternehmenskommunikation dienen, die Fehlinformationen, Falschinformationen und schädigende Informationen umfasst. ${ }^{1}$

Unter Fehlinformationen (Misinformation) werden Informationen verstanden, die faktisch nicht der Wahrheit entsprechen, jedoch vom Sender, der die Information verbreitet, für wahr gehalten werden (Wardle \& Derakhshan, 2018). Dabei kann es sich beispielsweise auch um irrelevante, ungenaue, vage, unsichere, mehrdeutige oder möglicherweise unvollständige Informationen handeln (Karlova \& Fisher, 2012). Möchte beispielsweise ein Unternehmen (Sender) mit einer bewusst mehrdeutig formu-

1 Auch wenn wir uns der anhaltenden Debatte um die passenden Begriffsbezeichnungen bewusst sind, möchten wir in diesem Beitrag zur besseren Unterscheidbarkeit und Lesbarkeit deutsche Begrifflichkeiten anbieten. 
lierten Überschrift (Information) zum Lesen einer Pressemitteilung anregen, kann es unter Umständen passieren, dass der eigentliche Text die Erwartungen des Journalisten (Empfänger) nicht erfüllt, die durch die Überschrift eigentlich geweckt worden sind (vgl. Clickbaiting im Journalismus). Dieses einfache Beispiel zeigt nicht nur den schmalen Grat zwischen Information und Fehlinformation, sondern auch das komplexe Zusammenspiel zwischen Intention und Rezeption.

Im Gegensatz dazu bezeichnen Falschinformationen (Disinformation) faktisch nicht der Wahrheit entsprechende Informationen, die vom Sender in dessen Bewusstsein absichtlich verbreitet werden (Wardle \& Derakhshan, 2018). Charakteristisch für diese Form ist die Täuschungsabsicht des Senders - unabhängig von den Motiven oder den Funktionen der Täuschung (Thummes, 2013). Hierunter fallen beispielsweise manipulierte oder gefälschte Inhalte, die gezielt in die Irre führen sollen (Karlova \& Fisher, 2012). Aus Sicht der Unternehmenskommunikation kommt dieser Form eine besondere Rolle zu, denn das Wissen des Senders - also einzelner Personen, Gruppen oder des gesamten Unternehmens - um die Täuschung macht diesen explizit angreifbar, da - wie im Folgenden noch aufgezeigt wird - möglicherweise keine Akzeptanz für die bewusste Täuschung seitens des Getäuschten vorherrscht. In diesem Fall geht es dann vor allem darum, das Aufdeckungsrisiko abzuwägen (Thummes, 2013).

Als dritte Form beschreiben schädigende Informationen (Malinformation) der Wahrheit entsprechende Informationen, die jedoch von einem Sender gezielt zur Benachteiligung eines anderen Akteurs eingesetzt werden (Wardle \& Derakhshan, 2018). Diese Form kennzeichnet sich vor allem durch die ausgeprägte Schadensabsicht, die oftmals an normative Verstöße gekoppelt ist und über ein rein öffentliches Interesse am Thema hinausgeht (Wardle \& Derakhshan, 2018). Beispielsweise könnte ein Unternehmen im Krisenfall die Beteiligung oder Mitwisserschaft anderer Unternehmen bekanntgeben (zum Beispiel durch gezielte Leaks), um so im Sinne der Strategie des Scapegoating (Coombs, 2007) die mediale und öffentliche Aufmerksamkeit vom eigenen Unternehmen abzulenken.

Es sei betont, dass es sich bei der Unterscheidung von Fehlinformationen, Falschinformationen und schädigenden Informationen um eine idealtypische Abgrenzung handelt, deren Trennschärfe in den verschiedenen Phasen der Informationsverbreitung von der Kreation über die Produktion und Distribution bis hin zu der Reproduktion verwischen kann (Wardle \& Derakhshan, 2018). Wie bereits erwähnt, gilt es dabei vor allem Faktoren der Sender (zum Beispiel Motive, Zielgruppe), der Informationen selbst (zum Beispiel Dauer, Genauigkeit) und der Rezipienten (zum Beispiel 
Verarbeitung, Anschlusshandlung) zu berücksichtigen (Wardle \& Derakhshan, 2018). Zahlreiche Beispiele aus der Praxis zeigen, in welchem Spannungsfeld sich Fehlinformationen, Falschinformationen und schädigende Informationen befinden können, wenn es der Unternehmenskommunikation um das Erreichen von PR-Zielen geht. Die folgenden Fälle sollen verschiedene Szenarien exemplarisch illustrieren.

\section{Fallbeispiele aus der Kommunikationspraxis}

Der Mineralölkonzern BP geriet im Jahr 2010 im Zuge der Ölpest im Golf von Mexiko in die Schlagzeilen, welche durch den Brand und den Untergang der Bohrinsel Deepwater Horizon ausgelöst wurde. Zuvor waren bereits Vorfälle wie die Explosion einer Raffinerie in Texas medienwirksam geworden. Insbesondere in den anfänglichen Stadien der Krise setzte BP auf die Strategie, sich möglichst wenig zu den Vorfällen zu äußern (Marohn, 2010). Im Laufe der Krise tauchten jedoch zudem manipulierte Bilder auf der Website von BP auf, welche die Situation rund um die Ölpest verharmlosten. BP rügte daraufhin nach eigenen Angaben den Fotografen, der für die Anfertigung und die Bearbeitung der Bilder zuständig war (Schultz, 2010).

Anhand dieses Falls lassen sich zwei zentrale Herausforderungen herausstellen: Einerseits die Bedeutung von verschwiegenen Informationen und andererseits die Identifikation von Motiven. Insbesondere die klassische Unternehmenskommunikation beruht größtenteils auf Push-Kommunikation. Dies bedeutet im Rückschluss, dass relevante Stakeholder davon ausgehen, aktiv durch Unternehmen informiert zu werden. Insofern stellt sich die Frage, inwieweit das gezielte Verschweigen von Informationen genauso schadhaft ist wie die Verbreitung von fehlleitenden, falschen, erfundenen oder manipulierten Inhalten. Gleichermaßen ist die Bestimmung der Fehlerhaftigkeit von Unternehmensinhalten für objektive Beobachter beinahe unmöglich. War BP beispielsweise über die Manipulation der Pressefotos unterrichtet, wäre der PR-Abteilung hier eine Falschinformation im Sinne wissentlich und absichtlich veränderter Begebenheiten zu unterstellen. Nach eigenen Angaben wusste das Unternehmen jedoch nichts über die Bildmanipulation. Insofern müsste der Fall - wenn man dem Unternehmen glauben möchte - als Fehlinformation gewertet werden.

Doch auch abseits von Fällen mit gesamtgesellschaftlicher Tragweite bewegt sich die Unternehmenskommunikation oft auf einem schmalen Grat zwischen ausgeklügelter PR und Fehlleitung der Öffentlichkeit. So wurde 
dem kommerziellen Radiosender 89.0 RTL im Jahr 2013 vorgeworfen, mit einer Tank-Aktion seine Kunden gezielt in die Irre geführt zu haben. Im Zuge dieser Aktion hatte der Radiosender öffentlich beworben, dass an ausgewählten Tankstellen Benzin für 89 Cent pro Liter ausgegeben wird. Verschwiegen wurde dabei der Fakt, dass nur die ersten drei Fahrzeughalter zu diesem Preis tanken konnten. Auch die Teilnahmebedingungen des Gewinnspiels wurden zeitweise von der Website entfernt (Kern, 2013). Während den Radiosender 89.0 RTL für diese Form von Falschinformation ein Shitstorm in den sozialen Medien ereilte, wurde das US-amerikanische Unternehmen IHOP im Jahr 2018 für seine aufmerksamkeitserregende PR-Aktion gelobt. So benannte sich das Unternehmen zeitweise in IHOB um, also International House of Burgers anstelle von International House of Pancakes, um auf seine neu eingeführten Burger aufmerksam zu machen. Nach ein paar Wochen stellte das Unternehmen in den sozialen Medien richtig, dass es sich bei dieser Falschinformation nur um einen PR-Gag handelte und das Unternehmen weiterhin den Namen IHOP trägt (Kittermann, 2018). Bis dahin wurde die Namensänderung jedoch schon von zahlreichen etablierten Medien aufgegriffen. Sogar die Konkurrenz reagierte darauf, indem beispielsweise die Fast Food-Kette Burger King ihr Logo und ihren Namen auf den Social-Media-Kanälen in Pancake-King änderte (Taylor, 2018). Insofern sind in dieser Kampagne auch Züge von schädigenden Informationen zu finden, da imagegefährdende Reaktionen der Konkurrenten zumindest billigend in Kauf genommen wurden.

In beiden Fällen wurde über einen längeren Zeitraum keine Richtigstellung vorgenommen und es wurden Informationen aufgrund kommerzieller Ziele zurückgehalten. Die fehlende Transparenz dient dabei als Stilmittel, um Aufmerksamkeit zu erzeugen. Somit wird auch klar, dass in diesen Fällen davon auszugehen ist, dass die Informationen nicht unbewusst verbreitet wurden, sondern von intentionaler, strategischer Kommunikation auszugehen ist.

Eine Person oder ein Unternehmen kann jedoch nicht nur Urheber von »Fake News« sein, sondern auch Opfer. So war das Berliner Fintech Startup-Unternehmen Kontist im Jahr 2018 durch eine gefälschte E-Mail eines anonymen Absenders betroffen. In dieser E-Mail wurde unter dem Namen der Pressesprecherin glaubwürdig eine zwölf Millionen Dollar Finanzierung für das Fintech-Unternehmen angekündigt, was daraufhin auf anerkannten Branchenportalen aufgegriffen wurde. Vermutet wurde dabei ein gezielter Angriff durch schädigende Informationen, was womöglich die damals aktuellen Verhandlungen über eine Finanzierung torpedieren sollte (Dohms, 2018). Fragen der strategischen Reaktionen, das Eindämmen von 
Dynamisierungsprozessen und die gezielte Bekämpfung von schädlichen Informationen stellen demnach weitere wichtige Herausforderungen für die Unternehmenskommunikation dar.

Diese vier Beispiele aus der Kommunikationspraxis verdeutlichen, dass die Grenzziehung zwischen Wahrheit und Unwahrheit, PR-Gag und gezielter Fehlleitung mitunter schwierig sein kann. Dies gilt auch für Instanzen wie den DRPR, der als freiwilliges Organ der Selbstkontrolle zuletzt seine Richtlinien zur Bekämpfung des Phänomens »Fake News« verschärft hat (DRPR, 2017). Um noch weiter für die Typologisierung von Fehlinformationen, Falschinformationen und schädigenden Informationen zu sensibilisieren, lohnt sich ein Blick auf vergangene Sanktionen anhand von ausgewählten Fällen des DRPR. Dabei werden die Ratsbeschlüsse zwischen Januar 2010 und August 2019 näher betrachtet. Zentral für den vorliegenden Kontext sind Verstöße gegen die Abschnitte 9 und 10 des Deutschen Kommunikationskodexes, welche die Wahrhaftigkeit von Informationen adressieren. Zudem sind aufgrund der Manipulation von Informationen oder Kontext sowie der Schädigung des Rufes des Berufsfeldes mitunter die Fairness-, Transparenz-, Loyalitäts- und Professionalitätsgebote betroffen:

Tabelle 1: Ausgewählte Ratssprüche (Januar 2010 bis August 2019) des DRPR mit Bezug auf Wahrhaftigkeit von Informationen (Abschnitte 9 und 10 Deutscher Kommunikationskodex)

\begin{tabular}{|l|l|l|l|}
\hline $\begin{array}{l}\text { Empfänger, } \\
\text { Jahr }\end{array}$ & Sanktion & Vorwurf & Begründung \\
\hline $\begin{array}{l}\text { HeiScreen, } \\
2019\end{array}$ & Rüge & $\begin{array}{l}\text { Falschinformation: Marktreife ei- } \\
\text { nes Bluttests zur Krebsdiagnostik }\end{array}$ & $\begin{array}{l}\text { Unangemessenheit in Wortwahl, } \\
\text { Zeitpunkt und Format der Kampagne, } \\
\text { obwohl keine Marktreife vorliegt }\end{array}$ \\
\hline $\begin{array}{l}\text { Bundeswehr, } \\
2019\end{array}$ & Mahnung & $\begin{array}{l}\text { Falschinformation: Uniformver- } \\
\text { bot auf der re:publica 2018 }\end{array}$ & $\begin{array}{l}\text { Korrektur, keine direkte Nachweis- } \\
\text { barkeit des Verbots }\end{array}$ \\
\hline $\begin{array}{l}\text { Mattel, } \\
\text { DOJO, }\end{array} 2019$ & Rüge & $\begin{array}{l}\text { Falschinformation: } \\
\text { Kampagne } \text { BScrabble heißt jetzt } \\
\text { Buchstaben-YOLO« }\end{array}$ & $\begin{array}{l}\text { keit trotz humorvoller Intention und } \\
\text { Kommunikation }\end{array}$ \\
\hline $\begin{array}{l}\text { Savedroid, } \\
2018\end{array}$ & Rüge & $\begin{array}{l}\text { Falschinformation/Verstoß gegen } \\
\text { Loyalitätsgebot: Irreführung auf } \\
\text { der Website }\end{array}$ & $\begin{array}{l}\text { Irreführung der Öffentlichkeit trotz } \\
\text { späterer Korrektur }\end{array}$ \\
\hline $\begin{array}{l}\text { Elias Walser, } \\
\text { Alex Kröll, } \\
\text { Karl Royer, } \\
2017\end{array}$ & Rüge & $\begin{array}{l}\text { Falschinformation/Verstoß gegen } \\
\text { bransparenz- und Loyalitätsge- } \\
\text { Seefeld }\end{array}$ & $\begin{array}{l}\text { ten, verzögerte Korrektur, Schädi- } \\
\text { gung des Ansehens des Berufsfeldes } \\
\text { bewaupe von }\end{array}$ \\
\hline
\end{tabular}




\begin{tabular}{|l|l|l|l|}
\hline $\begin{array}{l}\text { Maschmeyer } \\
\text { Group, }\end{array}$ & - & $\begin{array}{l}\text { Falschinformation/Verstoß gegen } \\
\text { Loyalitäts- und Professionalitäts- } \\
\text { gebot: Manipulation von Reputa- } \\
\text { tionsbeiträgen }\end{array}$ & aus Mangel an Beweisen eingestellt \\
\hline $\begin{array}{l}\text { ADAC, } \\
2015\end{array}$ & Rüge & $\begin{array}{l}\text { Falschinformation/Verstoß gegen } \\
\text { Loyalitäts- und Professionalitäts- } \\
\text { gebot: Wahlmanipulation bei } \\
\text { Verleihung des Gelben Engels }\end{array}$ & $\begin{array}{l}\text { keit } \\
\text { bewusste Täuschung der Öffentlich- }\end{array}$ \\
\hline $\begin{array}{l}\text { Deutsche } \\
\text { Börsen AG, }\end{array}$ & Rüge & $\begin{array}{l}\text { Falschinformation/Verstoß gegen } \\
\text { ordnungsgemäße Ad-Hoc-Publi- } \\
\text { zität: fehlerhafte Ad-Hoc-Mittei- } \\
\text { lung }\end{array}$ & $\begin{array}{l}\text { Fehlen von Vergleichszahlen aus der } \\
\text { Vherzustellen }\end{array}$ \\
\hline $\begin{array}{l}\text { Allianz, } \\
\text { Daimler, } \\
2014\end{array}$ & - & $\begin{array}{l}\text { Falschinformation: fehlerhafte } \\
\text { Ad-Hoc-Mitteilung: fehlende } \\
\text { Vergleichszahlen }\end{array}$ & $\begin{array}{l}\text { kein Verstoß, aber Vorschlag zur } \\
\text { Uberarbeitung der Richtlinien zur } \\
\text { Ad-Hoc-Publizität }\end{array}$ \\
\hline $\begin{array}{l}\text { Dr. Thomas } \\
\text { Raabe, } \\
2012\end{array}$ & Mahnung & $\begin{array}{l}\text { Falschinformation: Ausschluss } \\
\text { ziviler Opfer in Berichten zu Mi- } \\
\text { litäranschlag in Kunduz }\end{array}$ & $\begin{array}{l}\text { Irreführung der Öffentlichkeit, jedoch } \\
\text { nicht zweifelsfrei belegbar }\end{array}$ \\
\hline $\begin{array}{l}\text { Helmut Hoffer } \\
\text { von Ankers- } \\
\text { hoffen, }\end{array}$ & Rüge & $\begin{array}{l}\text { Falschinformation/Verstoß gegen } \\
\text { schung von Rezensionen }\end{array}$ & $\begin{array}{l}\text { Eindruck der Echtheit zur Steigerung } \\
\text { des Produktimages und der Verkaufs- } \\
\text { zahlen }\end{array}$ \\
\hline
\end{tabular}

Wie der Überblick über die Ratssprüche des DRPR in Tabelle 1 zeigt, wurden in den letzten Jahren zahlreiche Rügen in Bezug auf falsche, erfundene oder manipulierte Informationen ausgesprochen. Manche Verfahren endeten nur mit einer Mahnung oder wurden gänzlich eingestellt. Ein häufiges Problem ist dabei der Nachweis der Vorsätzlichkeit beziehungsweise die nicht zweifelsfrei prüfbare Beweislage. Durch zusätzliche Richtlinien, wie der Richtlinie zur Ad-Hoc-Publizität, der Richtlinie zur Online-PR oder der Richtlinie zu Social Bots wurden die Regelungen des DRPR zum Phänomen »Fake News« über die letzten Jahre hinweg noch weiter ausdifferenziert. Dies zeigt, dass sich die Sanktionierung von Verstößen im Bereich der Falschinformationen in den letzten Jahren immer weiter verschärfte, jedoch weiterhin Probleme hinsichtlich der Identifikation von Intentionen, damit auch der Beweisführung und der Abgrenzung zu andersartigen Verstößen, bestehen.

Eine öffentliche Rüge begründet sich in den aufgeführten Fällen in einer Irreführung der Öffentlichkeit durch schädliche Informationen, insbesondere wenn diese Irreführung bewusst und vor dem Hintergrund kommerzieller Ziele stattfand. Auch eine bewusste Täuschung von Journalisten wird daher geahndet. Der Terminus »Fake News « wurde in diesem Zusammenhang erstmals im Jahr 2018 in der Rüge gegenüber der Savedroid AG explizit als Grund für eine Sanktion des DRPR genannt. Zudem erarbeitete 
der DRPR in den Begründungen zu seinen Sanktionen mehrere Präzedenzurteile. Dabei wurde festgehalten, dass eine humorvolle Intention der PRMaßnahmen und eine spätere Korrektur der kommunizierten Information die Tatsache der Falschinformationen nicht rechtfertigt. Wie das Urteil zur Ad-Hoc-Publizität in Bezug auf die Deutsche Börsen AG zeigt, kann auch das Fehlen von Information als bewusste Irreführung eingestuft werden. Damit rücken weitere, im obigen Überblick nicht berücksichtigte Fälle fehlender und intransparenter Kommunikation in den Fokus. Dies sind beispielsweise Fälle, in denen Absender-, Auftraggeber- und Sponsoreninformationen verschleiert wurden, Angebote für Schleichwerbung und positive Berichterstattung gegenüber finanziellen Gefälligkeiten ausgetauscht oder verdeckte PR-Tätigkeiten ausgeführt wurden. Diese Grenzfälle von Falschinformationen erweitern die Ratssprüche um weitere Fälle, die ebenfalls mit Rügen und/oder Mahnungen geahndet wurden.

\section{Risiken und Implikationen}

Aus den bisher behandelten theoretischen Grundannahmen und erläuterten Praxisbeispielen lassen sich nun die zentralen Herausforderungen des Phänomens »Fake News« für die Unternehmenskommunikation in einer Risikomatrix systematisieren. Insgesamt bezieht sich die Matrix auf zwei Dimensionen: Auf der ersten Dimension stehen vom Unternehmen in das gesellschaftliche Umfeld ausgehenden Informationen (endogene Informationen) solche Informationen gegenüber, auf die ein Unternehmen reagieren muss (exogene Informationen). Auf der zweiten Dimension wird der Wahrheitsgehalt der Informationen erfasst. Dieser bewegt sich zwischen unintendiert falsch und intendiert falsch. Damit ergeben sich insgesamt vier Risikoszenarien: bewusste Falschinformationen, unbewusste Fehlinformationen, bewusst schädigende Informationen und unbewusst schädigende Informationen (vgl. Abbildung 1). 


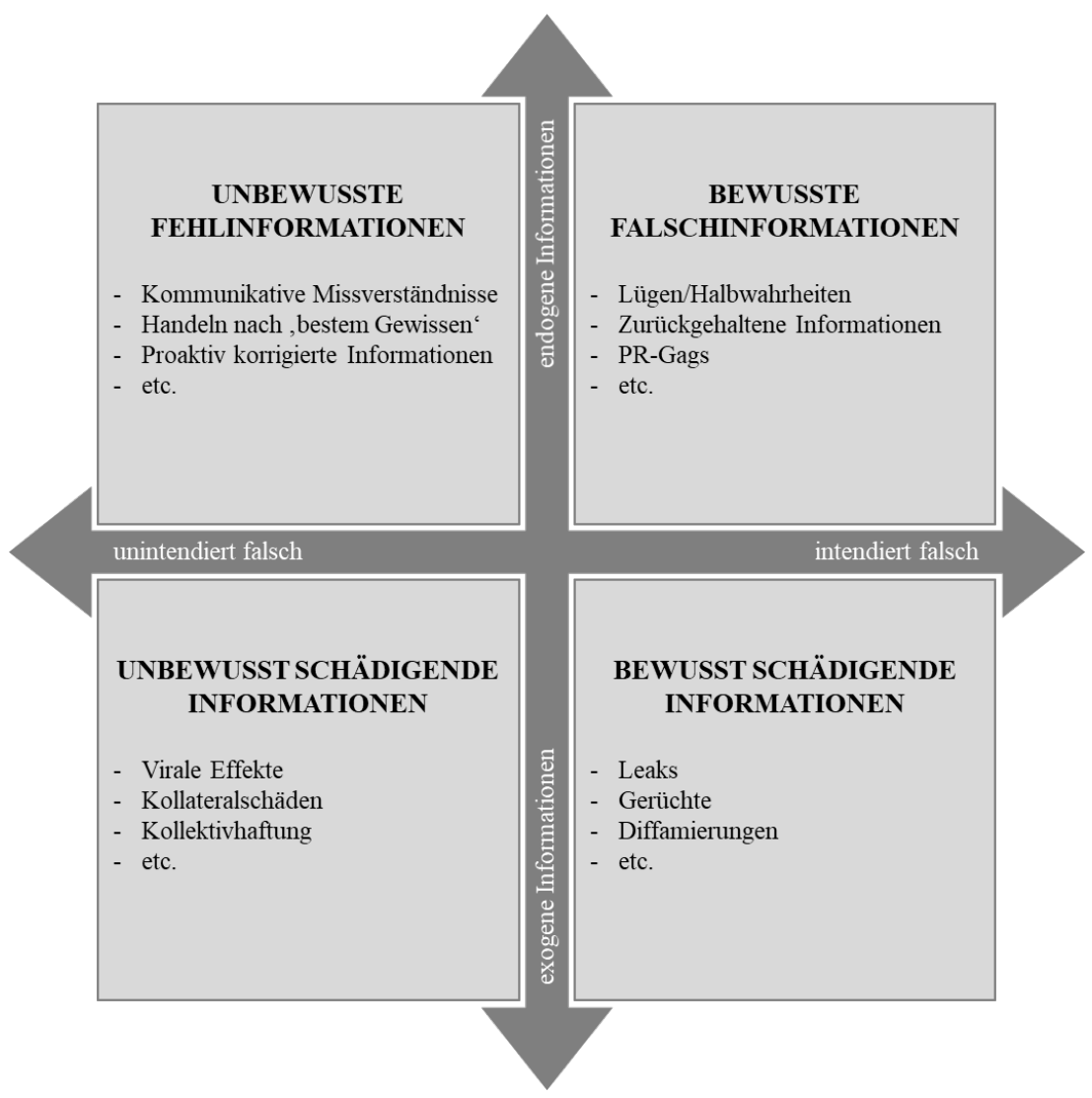

Abbildung 1: Risikomatrix des Phänomens »Fake News« in der Unternehmenskommunikation.

Das erste Risikoszenario stellen unbewusste Fehlinformationen dar. Bei Fehlinformationen werden fehlerhafte oder missverständliche Inhalte vom Unternehmen selbst verbreitet, ohne dass von den Kommunikatoren konkrete täuschende und schädigende Ziele verfolgt werden. Die Identifizierung von Fehlerquellen in Kommunikationsprozessen und entsprechende Intentionen sind per se eine Herausforderung der aktuellen »Fake News«Debatte, welche insbesondere auch die Regulierung betrifft. Exemplarisch dafür steht die Veröffentlichung manipulierter Bilder im Kontext der Ölpest im Golf von Mexiko durch BP. Das Unternehmen sah den Fehler dabei bei dem bearbeitenden Fotografen, da es nach eigenen Aussagen selbst nichts von der Manipulation wusste. Auch hier wird die Glaubwürdigkeit gegenüber dem Unternehmen und der Branche unterlaufen. Der Verlust von 
Glaubwürdigkeit durch irreführende Informationen - ob intendiert oder nicht - stellt dabei eine existenzielle Bedrohung für die PR dar (Jempson, 2005). Indem sie das Vertrauen in die Kommunikation mindern, untergraben Kommunikatoren die Glaubwürdigkeit aller Medien und werden somit zu ihren eigenen Feinden. Saubere Recherchen, Faktenchecks, Wahrheit und Transparenz des Berufsfeldes sind demnach zentrale Ziele, um die Platzierung und Weiterverbreitung von unbewussten Fehlinformationen einzudämmen.

$\mathrm{Zu}$ den bewussten Falschinformationen zählen solche Fälle, in denen Unternehmen selbst Falschinformationen als Strategie für die Erreichung ihrer Ziele einsetzen. Wie am Beispiel von IHOP und 89.0 RTL zu sehen war, werden hier Informationen verbreitet oder Informationen zurückgehalten, um Aufmerksamkeit zu erregen und relevante Stakeholder gezielt hinter das Licht zu führen. Bei dieser bewussten Irreführung entstehen zunächst Widersprüche mit den normativen Grundsätzen des PR-Berufsfeldes. Sie stellen Versuche dar, geltende Transparenz- und Wahrheitsgebote zu unterlaufen und gefährden somit auch den Ruf, die Professionalitätswahrnehmung und die Loyalität innerhalb der Kommunikationsbranche wie auch gegenüber der Öffentlichkeit. Dies heißt jedoch nicht, dass die strategische Kommunikation ihre Ziele nicht erreicht. Wie am Beispiel von IHOP gezeigt wurde, kann die gezielte Streuung von Falschinformation in Form von PRGags Aufmerksamkeit erzeugen und das Markenimage stärken. Generell ist Falschinformationen immer auch eine funktionale oder schützende Dimension inhärent (Mast, 2019; Thummes, 2013). Der strategische Einsatz von Falschinformationen ist somit eine hohe Kunst der PR und oszilliert im grundlegenden Spannungsfeld zwischen kommerziellen Interessen und normativen Kommunikationsgrundsätzen (Jempson, 2005).

Diesen beiden Feldern stehen Risikoszenarien gegenüber, die auf einer exogenen Informationsgrundlage im Sinne schädigender Informationen von außen beruhen. Unbewusst schädigende Informationen stellen dabei nicht nur eine Herausforderung für die Unternehmenskommunikation dar, sondern auch für alle anderen Gesellschaftsbereiche, die vom Phänomen »Fake News « betroffen sind. Risiken ergeben sich dabei insbesondere durch den Prozess der Verbreitung von Informationen und nicht durch die initial irreführende Information selbst (Giglietto, Iannelli, Valeriani \& Rossi, 2019). Dabei handelt es sich um sogenannte "propagation processes « (Giglietto et al., 2019, 14). Die unkontrollierbare Informationsdiffusion vor allem im Web 2.0 (zum Beispiel durch virale oder memetische Effekte) führt dazu, dass sich schädigende Informationen effizient in der Öffentlichkeit verbreiten und das Informationsökosystem gefährden (Johann \& Bülow, 2018). 
Wie eine aktuelle Studie zeigt, ist dabei das Glauben einer Nachricht getrennt von der Weiterverbreitung zu betrachten, denn das Teilen von Nachrichten ist sowohl in dem Bewusstsein der Falschheit einer Nachricht wie auch bei fehlendem Bewusstsein über die Falschheit beobachtbar (Wang, Margolin, Yuan \& Bazarova, 2019). Es handelt sich dabei also um Fehlinformationen, Falschinformationen oder schädigende Informationen, die meist durch eine Mischung aus Fehl- und Falschinformationen propagiert werden (Giglietto et al., 2019). Auch die Anschlusskommunikation etablierter Medien stellt in diesem Rahmen eine einzukalkulierende Gefahr dar, da die Schnelligkeit der Berichterstattung und unzureichende Faktenchecks den Verbreitungsprozess und die Glaubwürdigkeitskrise weiter vorantreiben (Jempson, 2005). Diese Propagierungsprozesse fordern von Unternehmen, dass sie sich auf grundlegende Werte ihres Berufsfeldes zurückbesinnen. In Fällen, bei denen Unternehmen - egal ob intendiert oder nicht intendiert - Urheber der falschen Informationen sind, ist es fraglich, ob die sozialen Medien zusätzlich als eine Art Brandbeschleuniger fungieren, auch wenn durch propagierende Falschinformationen eine Mitschuld bei externen Akteuren gesehen werden kann.

Das vierte Risikoszenario wird als bewusst schädigende Informationen bezeichnet. Hierzu zählen demnach gezielte Angriffe auf ein Unternehmen durch externe Akteure, welche schädigende Informationen als Strategie nutzen - etwa zur Diffamierung oder zur Verlagerung eines Themas. Diese Herausforderung ist vor allem für die PR nichts Neues und besteht bereits seit Beginn des Internets (Basso, 1997). Technologische Entwicklungen wie Social Bots führen jedoch dazu, dass gezielte Angriffe auf Unternehmen leichter durchführbar und für jeden zugänglich werden. Bei Social Bots handelt es sich um Fake-Profile in sozialen Medien, die mit dem Zweck erstellt werden, Falschinformationen in einem Netzwerk zu platzieren (Ferrara, Varol, Davis, Menczer \& Flammini, 2016). Wie am Beispiel des Fintech-Unternehmens Kontist aufgezeigt wurde, können Falschinformationen auf diese Weise sogar in den Geschäftsalltag eines Unternehmens eingreifen (Dohms, 2018). Dies birgt einerseits die Herausforderung, Urheber solcher Kampagnen in den anonymisierten Strukturen des Internets zu identifizieren und zu sanktionieren. Anderseits können auch hier wiederum Brandbeschleunigereffekte dazu führen, dass die Reputation eines Unternehmens Schaden nimmt und relevante Stakeholder in die Irre geführt werden. 
Fazit

Insgesamt ist festzuhalten, dass das Phänomen »Fake News« keine neue Herausforderung für die Unternehmenskommunikation darstellt, sondern alte Dilemmata der PR kondensiert. Schon lange vor der Etablierung der sozialen Medien und der Geburt des Begriffes »Fake News« war vor allem die PR mit Fragen der Wahrhaftigkeit von Informationen beschäftigt und wird dies auch weiterhin bleiben. Die zunehmende Verbreitungsgeschwindigkeit von Informationen über die sozialen Medien sowie das erodierende Vertrauen in Teilen der Gesellschaft verstärken jedoch Symptome und Wirkungen von Falschinformationen, was besondere Aufmerksamkeit seitens der PR verdient.

Der Begriff »Fake News« bleibt letztendlich nur schwer zu vereinheitlichen, da er zu einem höchst politisierten Buzzword avanciert ist (Brummette et al., 2018; Tandoc et al., 2018). Aus diesem Grund schlägt der vorliegende Beitrag speziell für den Kontext der Unternehmenskommunikation eine mehrdimensionale Betrachtungsweise auf das Phänomen vor, die sich durch eine Risikomatrix systematisieren lässt. Zwischen den verschiedenen Szenarien bestehen durchaus starke Spannungen wie auch Zusammenhänge, welche die Relevanz von Fehlinformationen, Falschinformationen und schädigenden Informationen endogener und exogener Art für die Unternehmenskommunikation aufzeigen.

Dieser Beitrag hat für einen ersten Systematisierungsversuch des Phänomens »Fake News« im Kontext der Unternehmenskommunikation sein Augenmerk vor allem auf die Makro- und Mesoebene gerichtet. Die Ausführungen haben jedoch auch gezeigt, dass die Identifikation und der Nachweis von Falschinformationen nach wie vor eine der größten Herausforderungen für die Kommunikationspraxis darstellen. Aktuelle Studien zeigen sogar, dass nur wenige Organisationen formale Richtlinien und Routinen zur Erkennung von Falschinformationen implementiert haben und sich überwiegend auf die Kompetenzen und Erfahrungen individueller Mitarbeiter verlassen (Meng et al., 2019; Zerfaß et al., 2018). In der weiteren Beschäftigung mit dieser Thematik sollte daher auch die Mikroebene mit besonderem Blick auf die Identifikation, Bewältigung und Prävention von Falschinformationen stärker berücksichtigt werden. 


\section{Literatur}

Allcott, Hunt \& Gentzkow, Matthew (2017). Social Media and Fake News in the 2016 Election. Journal of Economic Perspectives, 31(2), 211-236. DOI: 1257/jep.31.2.211.

Arendt, Florian; Haim, Mario \& Beck, Julia (2019). Fake News, Warnhinweise und perzipierter Wahrheitsgehalt. Zur unterschiedlichen Anfälligkeit für Falschmeldungen in Abhängigkeit von der politischen Orientierung. Publizistik, 64(2), 181-204. DOI: $10.1007 / \mathrm{s} 11616-019-00484-4$.

Baerns, Barbara (1985). Öffentlichkeitsarbeit oder Journalismus. Zum Einfluß im Mediensystem. Köln: Wissenschaft und Politik.

Basso, Joseph (1997). How Public Relations Professionals are Managing the Potential for Sabotage, Rumors, and Misinformation Disseminated via the Internet by Computer Hackers. IEEE Transactions on Professional Communication, 40(1), 28-33. DOI: $10.1109 / 47.557516$.

Bentele, Günter; Liebert, Tobias \& Seeling, Stefan (1997). Von der Determination zur Intereffikation. Ein integriertes Modell zum Verhältnis von Public Relations und Journalismus. In: Bentele, G. \& M. Haller (Hrsg.), Aktuelle Entstehung von Öffentlichkeit. Akteure - Strukturen - Veränderungen (225-250). Konstanz: UVK.

Brummette, John; DiStaso, Marcia; Vafeiadis, Michali \& Messner, Marcus (2018). Read All About It. The Politicization of »Fake News« on Twitter. Journalism \& Mass Communication Quarterly, 95(2), 497-517. DOI: 10.1177/1077699018769906.

Coombs, W. Timothy (2007). Protecting Organization Reputations During a Crisis. The Development and Application of Situational Crisis Communication Theory. Corporate Reputation Review, 10(3), 163-176. DOI: 10.1057/palgrave.crr.1550049.

Corner, John (2017). Fake News, Post-Truth and Media-Political Change. Media, Culture \& Society, 39(7), 1100-1107. DOI: 10.1177/0163443717726743.

Dohms, Heinz-Roger (2018). Berliner Fintech Kontist wird Opfer von Fake-News-Attacke. Abgerufen von https://www.gruenderszene.de/fintech/berliner-fintech-kontistwird-opfer-von-fake-news-attacke.

DRPR (2017). Presseinformation des Deutschen Rats für Public Relations (DRPR). DRPR verabschiedet neu aufgelegte Online-Richtlinie. Abgerufen von http://drpronline.de/wp-content/uploads/2017/10/PM_DRPR_Online-Richtlinie_171002.pdf.

Dulek, Ronald E. \& Campbell, Kim S. (2015). On the Dark Side of Strategic Communication. International Journal of Business Communication, 52(1), 122-142. DOI: $0.1177 / 2329488414560107$.

Edelman (2019). 2019 Edelman Trust Barometer. Global Report. Abgerufen von https://www.edelman.de/trust-2019.

European Association of Communication Directors (EACD) (2017). Manifesto Against Fake News. Abgerufen von https:/www.eacd-online.eu/insights/blog/manifestoagainst-fake-news.

Farsetta, Diane \& Price, Daniel (2006). »Fake TV News«. Widespread and Undisclosed. Madison, WI: Center for Media and Democracy. 
Ferrara, Emilio; Varol, Onur; Davis, Clayton; Menczer, Filippo \& Flammini, Alessandro (2016). The Rise of Social Bots. Communications of the ACM, 59(7), 96-104. DOI: $10.1145 / 2818717$.

FleishmanHillard (2018). Authenticity Gap Report. Authenticity in an uncertain world. How to build trust and bridge the gap between what people expect and what they experience. Abgerufen von http://www.authenticity-gap.de.

Gelfert, Axel (2018). Fake News. A Definition. Informal Logic, 38(1), 84-117. DOI: 10.22329/il.v38i1.5068.

Giglietto, Fabio; Iannelli, Laura; Valeriani, Augusto \& Rossi, Luca (2019). »Fake news« is the Invention of a Liar. How False Information Circulates Within the Hybrid News System. Current Sociology, 67(4), 625-642. DOI: 10.1177/0011392119837536.

Gunther, Richard; Beck, Paul A. \& Nisbet, Erik C. (2019). »Fake News« and the Defection of 2012 Obama Voters in the 2016 Presidential Election. Electoral Studies. DOI: 10.1016/j.electstud.2019.03.006.

Habermas, Jürgen (1990). Strukturwandel der Öffentlichkeit. Untersuchungen zu einer Kategorie der bürgerlichen Gesellschaft. Frankfurt a. M.: Suhrkamp.

Hoffjann, Olaf (2009). Public Relations als Differenzmanagement von externer Kontextsteuerung und unternehmerischer Selbststeuerung. Medien- \& Kommunikationswissenschaft, 57(3), 299-315. DOI: 10.5771/1615-634x-2009-3-299.

Ireton, Cherilyn \& Posetti, Julie (Hrsg.) (2018). Journalism, »Fake News« \& Disinformation. Handbook for Journalism Education and Training. Paris: United Nations Edu-cational, Scientific and Cultural Organization.

Jackob, Nikolaus; Schultz, Tanjev; Jakobs, Ilka; Ziegele, Marc; Quiring, Oliver \& Schemer, Christian (2019). Medienvertrauen im Zeitalter der Polarisierung. Media Perspektiven, 5, 210-220.

Jempson, Mike (2005). Spinners or Sinners? PR, Journalists and Public Trust. Journal of Communication Management, 9(3), 267-276. DOI: 10.1108/13632540510621542.

Johann, Michael (2020). Dialogorientierte Unternehmenskommunikation in den sozialen Medien. Langfristige Entwicklungen und Einflüsse auf organisationale Beziehungen. Wiesbaden: Springer VS. [im Erscheinen].

Johann, Michael \& Bülow, Lars (2018). Die Verbreitung von Internet-Memes. Empirische Befunde zur Diffusion von Bild-Sprache-Texten in den sozialen Medien. kommunikation@gesellschaft,19(1),1-24.

Karlova, Natascha A. \& Fisher, Karen E. (2012). »Plz RT«. A Social Diffusion Model of Misinformation and Disinformation for Understanding Human Information Behaviour. Proceedings of the ISIC, Japan, 18, 1-17.

Kern, Ekki (2013). Nach Shitstorm. 89.0 RTL stoppt 89-Cent-Tank-Aktion. Abgerufen von https://www.radiowatcher.de/2013/09/05/89-0-rtl-tank-aktion-89cent-shitstorm/ 2586.

Kirf, Bodo (2018). Unternehmenskommunikation in Zeiten digitaler Transformation. In: Kirf, B.; K.-N. Eicke \& S. Schömburg (Hrsg.), Unternehmenskommunikation im Zeitalter der digitalen Transformation (1-54). Wiesbaden: Springer Gabler. DOI: 10.1007/978-3-658-15364-9_1. 
Kitterman, Ted (2018). »IHOb«Is No More. IHOP Cops to Name Change Stunt. Abgerufen von https://www.prdaily.com/ihob-is-no-more-ihop-cops-to-name-changestunt.

Kohring, Matthias \& Zimmermann, Fabian (2019). Die wissenschaftliche Beobachtung aktueller Desinformation. Eine Entgegnung auf Armin Scholls und Julia Völkers Anmerkungen in »Fake News, aktuelle Desinformationen und das Problem der Systematisierung « in M\&K 2/2019. Medien \& Kommunikationswissenschaft, 67(3), 319325. DOI: $10.5771 / 1615-634 x-2019-3-319$.

Marohn, Anna (2010). Nur zugeben, was nicht zu leugnen ist. Abgerufen von https://www.zeit.de/2010/24/BP-PR-Desaster.

Mast, Claudia (2019). Unternehmenskommunikation. München: UVK.

Meng, Juan; Reber, Bryan H.; Berger, Bruce K.; Gower, Karla K. \& Zerfaß, Ansgar (2019). North American Communication Monitor 2018-2019. Tracking Trends in Fake News, Issues Management, Leadership Performance, Work Stress, Social Media Skills, Job Satisfaction and Work Environment. Tuscaloosa, AL: The Plank Center for Leadership in Public Relations.

Merten, Klaus (2010). Ethik der PR. Ethik oder PR für PR. In: Hömberg, W.; D. Hahn \& T. B. Schaffer (Hrsg.), Kommunikation und Verständigung. Theorie - Empirie Praxis (95-118). Wiesbaden: Springer VS. DOI: 10.1007/978-3-531-92281-2_6.

Müller, Philipp \& Denner, Nora (2017). Was tun gegen »Fake News«? Eine Analyse anhand der Entstehungsbedingungen und Wirkweisen gezielter Falschmeldungen im Internet. Potsdam-Babelsberg: Friedrich-Naumann-Stiftung für die Freiheit.

Röttger, Ulrike; Preuße, Joachim \& Schmitt, Jana (2014). Grundlagen der Public Relations. Eine kommunikationswissenschaftliche Einführung. Wiesbaden: Springer VS. DOI: 10.1007/ 978-3-531-19965-8.

Scholl, Armin \& Völker, Julia (2019). Fake News, aktuelle Desinformationen und das Problem der Systematisierung. Anmerkungen zum Aufsatz von Fabian Zimmermann \& Matthias Kohring: »Fake News« als aktuelle Desinformation - systematische Bestimmung eines heterogenen Begriffs « in M\&K 4/2018. Medien \& Kommunikationswissenschaft, 67(2), 206-214. DOI: 10.5771/1615-634x-2019-2-206.

Schultz, Stefan (2010). PR-Panne. BP blamiert sich mit manipuliertem Foto von ÖlpestZentrum. Abgerufen von https://www.spiegel.de/wirtschaft/unternehmen/pr-pannebp-blamiert-sich-mit-manipuliertem-foto-von-oelpest-zentrum-a-707643.html.

Tandoc, Edson C.; Lim, Zheng Wei \& Ling, Richard (2018). Defining »Fake News«. A Typology of Scholarly Definitions. Digital Journalism, 6(2), 137-153. DOI: 10.1080/21670811.2017.1360143.

Taylor, Kate (2018). Burger King Has Changed its Name to »Pancake King« to Mock IHOP's Rebrand as IHOb. Abgerufen von https://www.businessinsider.de/burgerking-renamed-pancake-king-to-mock-ihop-2018-6.

Thummes, Kerstin (2013). Täuschung in der strategischen Kommunikation. Eine kommunikationswissenschaftliche Analyse. Wiesbaden: Springer VS. DOI: 10.1007/9783-658-00530-6. 
Thummes, Kerstin (2016). Zwischen Schutz und Manipulation. Eine Befragung zur Akzeptanz von Täuschungen durch Unternehmen. Medien \& Kommunikationswissenschaft, 64(3), 359-378. DOI: 10.5771/1615-634x-2016-3-359.

Wang, Luping; Margolin, Drew; Yuan, Connie \& Bazarova, Natalya (2019). Sharing Fake News on Social Media. Understanding the Relationship Between Believe and Share. Paper präsentiert auf der ICA Jahreskonferenz 2019, Washington, USA.

Wardle, Claire \& Derakhshan, Hossein (2018). Thinking About »Information Disorder«. Formats of Misinformation, Disinformation, and Mal-information. In: Ireton, C. \& J. Posetti (Hrsg.), Journalism, »Fake News« \& Disinformation. Handbookfor Journalism Education and Training (44-56). Paris: United Nations Educational, Scientific and Cultural Organization.

Zerfaß, Ansgar (2014). Unternehmenskommunikation und Kommunikationsmanagement. Strategie, Management und Controlling. In: Zerfaß, A. \& M. Piwinger (Hrsg.), Handbuch Unternehmenskommunikation. Strategie - Management - Wertschöpfung (21-79). Wiesbaden: Springer Gabler. DOI: 10.1007/978-3-8349-4543-3_2.

Zerfaß, Ansgar; Tench, Ralph; Verhoeven, Piet; Verčič, Dejan \& Moreno, Ángeles (2018). European Communication Monitor 2018. Strategic Communication and the Challenges of Fake News, Trust, Leadership, Work Stress and Job Satisfaction. Results of a Survey in 48 Countries. Berlin: Quadriga Media.

Ziegele, Marc; Schultz, Tanjev; Jackob, Nikolaus; Granow, Viola; Quiring, Oliver \& Schemer, Christian (2018). Lügenpresse-Hysterie ebbt ab. Mainzer Langzeitstudie »Medienvertrauen«. Media Perspektiven, 4, 150-162.

Zimmermann, Fabian \& Kohring, Matthias (2018). »Fake News« als aktuelle Desinformation. Systematische Bestimmung eines heterogenen Begriffs. Medien \& Kommunikationswissenschaft, 66(4), 526-541. DOI: 10.5771/1615-634x-2018-4-526. 